\title{
Quantifying differences between primary cortical areas in humans based on laminar profiles in in vivo MRI data
}

\author{
Juliane Dinse ${ }^{1,2}$, Pablo Martin ${ }^{1}$, Andreas Schäfer ${ }^{1}$, Stefan Geyer $^{1}$, \\ Robert Turner ${ }^{1}$, Pierre-Louis Bazin ${ }^{1}$ \\ ${ }^{1}$ Max Planck Institute of Cognitive and Brain Science, Stephanstr. 1a, 04103 Leipzig \\ ${ }^{2}$ Simulation and Graphics Department, Faculty of Computer Science, \\ Otto-von-Guericke University Magdeburg \\ dinse@cbs.mpg.de
}

\begin{abstract}
This paper presents an approach for mapping the human cortical architecture in vivo based on quantitative MRI indices of myelin. We automatically construct laminar profiles in several primary cortical areas and investigate different sampling strategies. The results demonstrate that our method is able to distinguish these areas at specific cortical depths.
\end{abstract}

\section{Introduction}

The human brain is a complex organ, including a highly convoluted cortex. Functional activity occur in a 2-5 mm thin sheet of neurons along the cortical surface, organized in six layers. The relative thickness of these layers changes in different areas of the brain which corresponds to different functional roles. In the early $20^{\text {th }}$ century, neuroanatomists started to investigate areal differences [1], deriving comprehensive measures of the cortical laminar pattern. The myeloarchitecture mappings of the Vogts' described the anatomical features associated with the myelin sheaths of neuronal axons and included over 200 cortical areas. However, their findings were defined on subjective and qualitative measures on two-dimensional stained tissue sections of post-mortem brains.

Magnetic resonance imaging (MRI) enables scientists to measure structural and functional features in vivo. With an increased resolution and sensitivity, methods have evolved which are able to map the organization of the cortex by imaging the myelin content. Geyer et al. demonstrated that 7 Tesla MRI reveals local cortical differences in quantitative T1 images and can precisely depict the cortical boundaries [2]. Glasser and van Essen published a new method based on myelin content, as revealed by T1-weighted and T2-weighted MRI [3]. Clare and Bridge have investigated how reliably cortical areas and boundaries can be detected using MRI [4]. But to what extend are myelin-related laminar profiles in MR data specifiable between primary cortical areas?

To our knowledge, the approach presented here is the first ever published comparison of quantitative myelin-related laminar profiles between primary areas (motor, somatosensory and visual), based on in vivo MRI. We investigate 

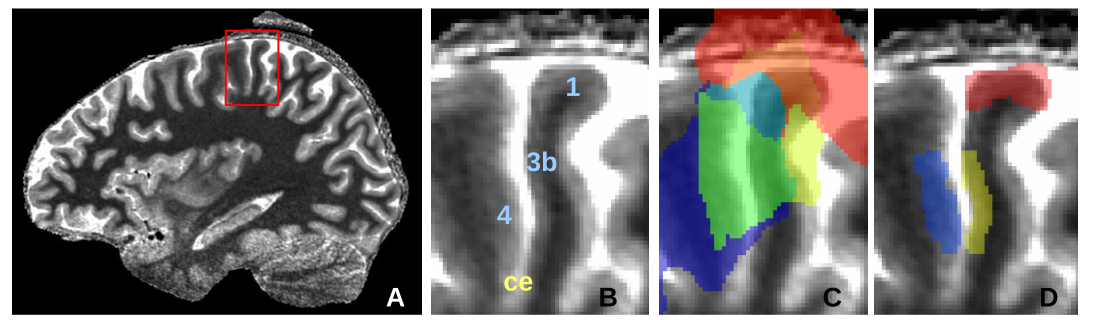

Fig. 1. A quantitative T1 map of the brain (A) with the areas in focus (B) in the central sulcus (ce). The probabilistic samples (C) overlap neighboring regions, whereas the manual samples (D) are within microstructural borders.

and compare two different methods of obtaining samples in regions-of-interest (ROIs): probabilistic atlases based on cytoarchitectonic data of post-mortem brains and manually sampling voxels in regions defined by macro-anatomical landmarks. Our results allow for a first quantitative comparison to the myeloarchitecture of the cortex and outline new possibilities of incorporating such information into new parcellation approaches. A proper mapping of the cortical organization will provide enhanced models of the human cortex for neuroimaging, especially if myelination patterns can be reproduced robustly in individual brains.

\section{Materials and Methods}

Six MRI data sets with $0.7 \mathrm{~mm}^{3}$ resolution have been acquired according to the MP2RAGE imaging sequence [5,6]. The data is registered to the Montreal's Neurological Institute (MNI) brain space to preserve cortical geometry and resampled to $0.4 \mathrm{~mm}^{3}$ resolution. The rigid registration includes six degrees of freedom and is optimized using a cost function of normalized mutual information. The cortex is extracted using in-house software [7] integrated in the MIPAV framework (http://mipav.cit.nih.gov/). The boundaries $\varphi_{G W}$ between gray matter (GM) and white matter (WM) and $\varphi_{G C}$ between GM and cerebro-spinal fluid (CSF) estimated during the segmentation are represented as level set surfaces [8] and used to estimate $l$ equi-distant laminae in-between $(l=20)$ with the following differential equation

$$
\frac{\partial \varphi_{d}}{\partial t}+\left(\varphi_{d}-\left((1-\rho) \varphi_{G W}-\rho \varphi_{G C}\right)\right)\left|\nabla \varphi_{d}\right|=\epsilon \kappa\left|\nabla \varphi_{d}\right|
$$

where $(1-\rho) \varphi_{G W}-\rho \varphi_{G C}$ is the target laminae as a weighted average function of $\rho \in[0,1]$ The cortical depth is computed at each point depending on the desired lamina and local curvatures. $\epsilon \kappa\left|\nabla \varphi_{d}\right|$ is a level set regularization term which avoids shocks and smooths the laminae. The real cortical layers are 
different from our estimated laminae. The relative thickness of the six cortical layers varies due to curvature, thus compensating for the folding. Our method only provides a coordinate system for measuring cortical depth. Based on the set of the equi-distant laminae $\left\{\varphi_{d}\right\}$, orthogonal profile curves can be generated as follows: from any starting location $x$, the projection onto the closest lamina $\varphi_{d}$ is obtained as

$$
x_{d}=x-\varphi_{d}(x) \frac{\nabla \varphi_{d}(x)}{\left|\nabla \varphi_{d}(x)\right|}
$$

and $x_{d}$ is projected onto the next closest lamina, until a curved 3D profile is generated that intersects all the layered laminae.

The constructed profiles are the basis of this work. We select four ROIs of primary cortical areas: Brodmann Area BA 1 and BA 3b (somatosensory cortex), BA 4 (motor cortex) and BA 17 (visual cortex) within the left hemisphere. For the atlas-based sampling we use the probabilities given in the Jülich atlas [9]. We threshold the individual ROIs at the probability $p=25 \%$ in order to ensure a large enough sample size. The manually sampling on individual brains is guided by macro-anatomical landmarks which in primary areas show good correlation with microstructural borders. The profile analysis is done in MATLAB (MathWorks, Inc).

For each subject and each ROI, we calculate an average profile consisting of mean and standard deviation per lamina. To do so, the intensity histogram per lamina in individual subjects and ROIs is computed and a Gaussian curve is fitted to it based on a Maximum-Likelihood approach. The mean and standard deviation are derived from the distribution curve. For comparison among the overall intensities in the cortex, we compute histograms, their means and standard deviations for the entire hemisphere. We calculate the correlation coefficients of the profile for each ROI between all subjects, for each of the two different sampling methods, to ensure that the shapes follow the same pattern. Furthermore, we calculate the difference between the cortex and each ROI for each subject. Based on these difference measures, the mean and standard deviation are computed again. In order to verify the significance of the results, and to demonstrate that profiles can be distinguished at specific cortical depths, a z-test was carried out per lamina between 0.25 to 0.9 cortical depth. The range of the cortical depth has been limited due to partial volume effects at the WM/GM and GM/CSF interfaces. Based on the test outcome, a rejection rate $R=$ rejected cases/all tested cases is calculated. Finally, the mean and standard deviations of the differences of manual sampled voxels are graphed in order to visually emphasize their areal distinction.

\section{Results}

The focus in the following sections is on BA 1, 3b and 4. However, BA 17 has been processed in the same way. The results are similar, but have been omitted due to 

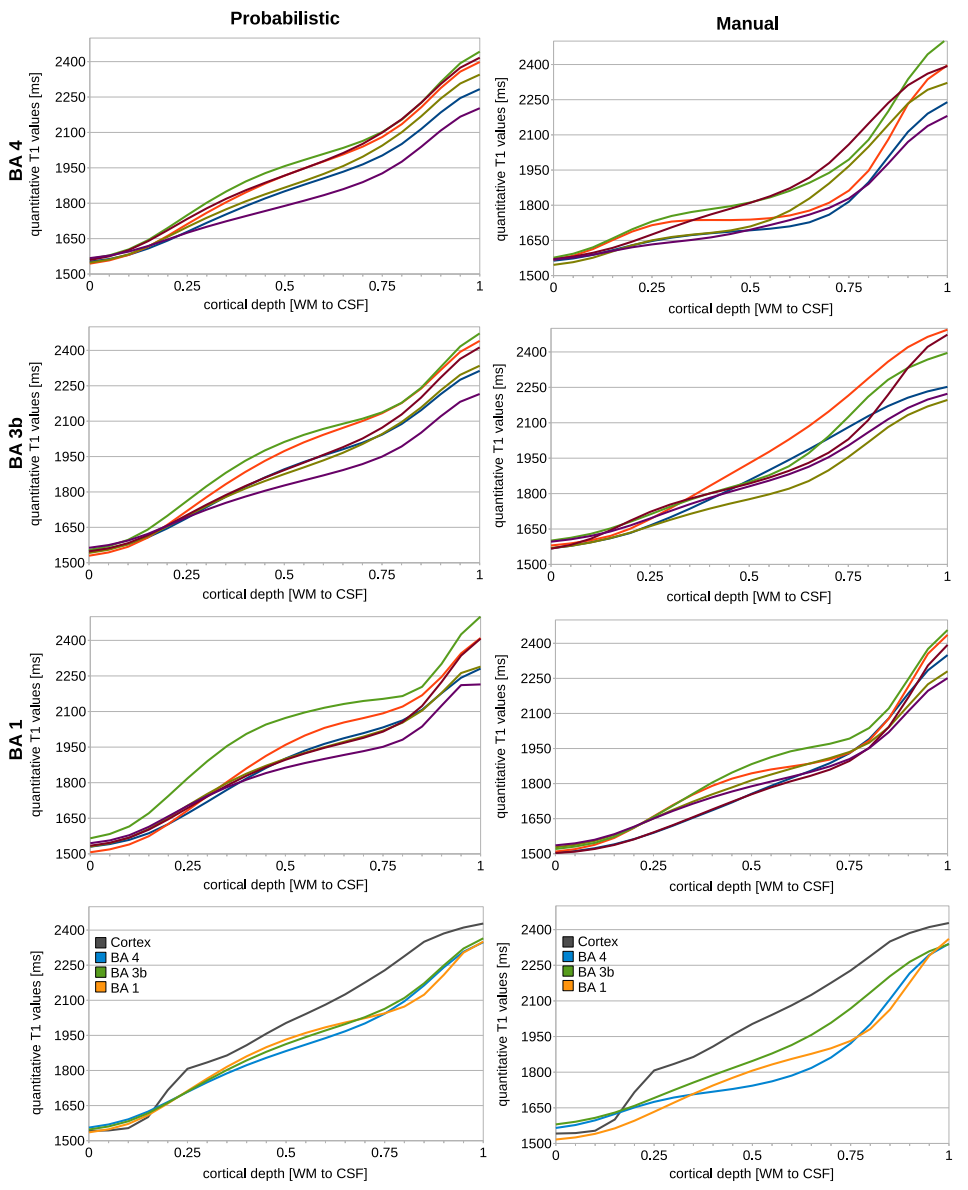

Fig. 2. Average profiles of primary cortical areas in focus (rows 1-3) each calculated in six subjects. The bottom row plots the group average of the individual ROIs according to the groups' average profile of the cortex.

space limitations. Fig. 1 depicts the different sampling methods in an individual brain. BA 1, 3b and 4 are anatomically in close vicinity, but related to different function, and should have different myelin-related profiles. In contrast to the manually sampled elements guided by macro-anatomical landmarks, Fig. 1 shows that samples of the probabilistic atlas overlap to a high degree. They also sample in neighboring regions. Fig. 2 (rows 1-3) shows the average profiles of all subjects in BA 1, 3b and 4 for both sampling strategies. All subjects have fairly consistent profiles, especially for the manually sampled ROIs. The profile shape in manually sampled ROIs are descriptive, in particular in BA 1. The correlation calculation for each ROI in both samplings always resulted in $r=1$ and $p=0$, meaning the profiles correlate well between subjects. When averages are grouped over the six 
subjects in each ROI and the cortex (see Fig. 2, bottom row), it becomes obvious that the mean profiles in the individual ROIs of the manual sampling are much more distinct than those derived from the probabilistic profiles. After calculating the mean and standard deviation of the differences between the cortex and each ROI, both methods show some strong dissimilarities between primary areas and the cortex as a whole. In the manual sampling, all ROIs are also distinct from each other (see Fig. 3) whereas the probabilistically sampled ROIs were very similar. The mean differences from the manual sampling are larger, standard deviations are smaller, or at least comparable. BA 4 and BA 3b follow the same profile pattern (location of increase and decrease of slope) with BA 4 always having larger differences between 0.3 to 0.8 of cortical depth. Using manual sampling this difference is more than doubled. BA 1 is also distinguishable from all other ROIs. The z-test for probabilistic sampling showed that on average in $R=41.67 \%$ of compared cases the profiles are distinguishable between each other (min: BA 4: $28.57 \%$, max: BA 1: $54.76 \%$ ). The z-test results for the manual samples were much higher with $R=80.16 \%$ on average (min: BA 1 and BA $3 \mathrm{~b}$ : $78.57 \%$, max: BA 4: $83.33 \%$ ). In former studies, we saw that profile shapes changed with less than 1000 samples. In this study sample sizes for probabilistic atlases are on average: 20.848 (BA 1), 85.311 (BA 3b) and 79.305 (BA 4). The sizes for manual sampling are: $2.400,1.810$ and 2.660 , respectively.

\section{Discussion}

We have shown that it is possible to compare quantitative areal differences in laminar profiles of T1 between primary areas in in vivo MRI data of the human cortex. The profiles of the subjects in each ROI show excellent consistency. The averaged profiles in each ROI show good agreement with architectonic differences published by Vogt and Vogt [1]. BA 4 is highly myelinated with their Bands of Baillarger mainly obscured, thus having shorter T1 values in a larger range of cortical depth (Fig. 2). However, BA 3b shows Bands of Baillarger and their corresponding profiles show higher T1 values in mid cortical depth. Also, the relative thickness of myelinated layers is reflected in the profiles. In summary, our proposed quantification method is able to model myelination relations in primary areas.

However, in this first analysis there are some limitations. The resolution of the data analyzed is not yet sufficient to reveal the full microstructure of the cortex. Some important features of the actual cortical layering are not represented in the MRI data. Improved resolution will make it easier to deal with partial voluming effects, especially in regions with highly folded structures (BA 1 and BA 17). Furthermore, the equi-distant laminae used do not confirm to the actual cortical layering and thus will sometimes cross cortical layers. A more accurate volumepreserving model which incorporates cortical curvature is under investigation [10]. The problem of correct sampling is hard to resolve. Probabilistic ROIs from the Jülich atlas provide only an approximate localization due to individual variations and include samples from neighboring areas. In large areas such as 


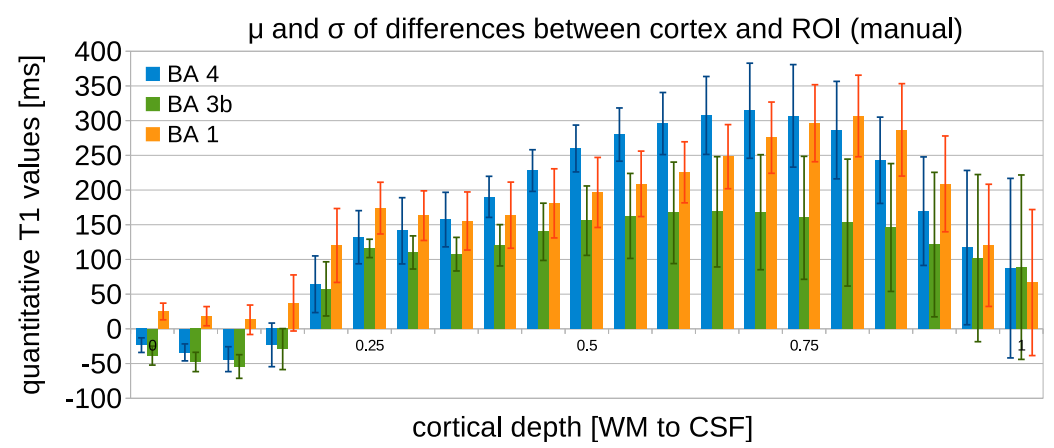

Fig. 3. Mean and standard deviation of the differences between the cortex and each ROI in all cortical depths.

BA 17, the effect can be small, but in most places it will distort the profile. For these reasons, it is desirable to have subject-specific definitions of the areas. This study shows that it is possible to derive a quantitative model which can be obtained with high-resolution quantitative MRI and analysis of cortical profile differences. More work is needed to refine such a model, extend it to more areas and to finally segment and classify the areas in new subjects.

\section{References}

1. Vogt C, Vogt O. Allgem. Ergebnisse unserer Hirnforschung. 1. Mitteilung. Ziele und Wege unserer Hirnforschung. J Psychol Neurol. 1919;25.

2. Geyer S, Weiss M, Reimann K, et al. Microstructural Parcellation of the Human Cerebral Cortex-From Brodmann's Post-Mortem Map to in vivo Mapping with High-Field Magnetic Resonance Imaging. Frontiers in Human Neuroscience. 2011;5.

3. Glasser MF, Van Essen DC. Mapping Human Cortical Areas In Vivo Based on Myelin Content as Revealed by T1-and T2-Weighted MRI. J Neurosci. 2011;31(32).

4. Clare S, Bridge H. Methodological issues relating to in vivo cortical myelography using MRI. Hum Brain Map. 2005;26(4).

5. Marques J, Kober T, Krueger G, et al. MP2RAGE, a self bias-field corrected sequence for improved segmentation and T1-mapping at high field. NeuroImage. 2010;49(2).

6. Hurley AC, Al-Radaideh A, Bai L, et al. Tailored RF pulse for magnetization inversion at ultrahigh field. MRM. 2010;63(1).

7. Bazin PL, Weiss M, Dinse J, et al. A computational pipeline for subject-specific, ultra-high resolution cortical analysis at 7 Tesla. Hum Brain Map. 2012;(abstract).

8. Sethian JA. Level set methods and fast marching methods: evolving interfaces in computational geometry, fluid mechanics, computer vision, and materials science. Cambridge University Press; 1999.

9. Eickhoff S, Stephan K, Mohlberg H, et al. A new SPM toolbox for combining probabilistic cytoarchitectonic maps and functional imaging data. NeuroImage. $2005 ; 25(4)$.

10. Waehnert M, Weiss M, Streicher M, et al. Do cortical layers conform to the Laplace equation? Hum Brain Map. 2012;(abstract). 